\title{
The burden of visceral leishmaniasis in Italy from 1982 to 2012: a retrospective analysis of the multi-annual epidemic that occurred from 1989 to 2009
}

M Gramiccia' A Scalone $^{1}$, T Di Muccio ${ }^{1}$, S Orsini ${ }^{1}$, E Fiorentino ${ }^{1}$, L Gradoni (luigi.gradoni@iss.it) ${ }^{1}$

1. Unit of Vector-borne Diseases and International Health, MIPI Department, Istituto Superiore di Sanità, Rome, Italy

Citation style for this article:

Gramiccia M, Scalone A, Di Muccio T, Orsini S, Fiorentino E, Gradoni L. The burden of visceral leishmaniasis in Italy from 1982 to 2012 : a retrospective analysis of the multi-annual epidemic that occurred from 1989 to 2009. Euro Surveill. 2013;18(29):pii=20535. Available online: http://www.eurosurveillance.org/ViewArticle. aspx?Articleld $=20535$

Article submitted on 10 August 2012/ published on 18 July 2013

Starting from 1989 Italy experienced an increase of visceral leishmaniasis (VL) cases over a baseline of 10 to 30 cases reported annually. The number of cases peaked in 2000 and 2004 with more than 200 cases/ year, and subsequently declined to reach on average one third of the 2000 peak value in the period after 2010. A retrospective analysis from 1982 to 2012 showed that the multi-annual epidemic consisted of major components including (i) an outbreak involving infants and immunocompetent adults in parts of the Campania region (southern peninsular Italy) and that appears to have declined naturally, (ii) a second outbreak affecting human immunodeficiency virus (HIV)-infected individuals throughout the country, that declined owing to the use of highly active antiretroviral therapies (HAART), (iii) a generalised increase of VL cases in immunocompetent individuals and patients affected by associated conditions other than HIV from endemic regions of peninsular and insular Italy (other than Campania), which was due to a geographical spreading of VL foci, with no major case-clusters or outbreak features. A minor component consisted in the appearance of a few autochthonous cases in formerly non-endemic areas, starting from the early 1990 . Epidemic determinants and reasons for VL decline in the Campania region remain largely unexplained, despite the information available on canine reservoir and phlebotomine vectors in Italy.

\section{Introduction}

Visceral leishmaniasis (VL) is a protozoan disease transmitted by phlebotomine sandflies and caused by members of the Leishmania donovani complex. The disease results from the systemic intracellular infection of the macrophage-rich organs. The incubation period is long (an average of 3-8 months, reaching up to >10 years in case of reactivation from latent infections) and the chronic appearance of signs and symptoms makes a clinical suspicion difficult in low or non-endemic areas. Epidemiological features and determinants of VL outbreaks are largely diverse. Epidemics of anthroponotic VL caused by L. donovani sensu stricto (a species recently introduced in the Mediterranean [1]) have long been known to occur as multi-annual waves with, as seen in outbreaks in India and East Africa, thousands of cases in wide areas, followed by interepidemic periods of five to 20 years [2-5]. On the other hand, zoonotic VL, a disease caused by L. infantum for which domestic dogs act as the main reservoir hosts, exhibits a typical pattern characterised by isolated or small localised clusters of cases (usually less than 10), with large epidemics uncommon in the Mediterranean area [6]. The first documented outbreak of zoonotic VL occurred within the 1971 to 1972 period, near Bologna, Italy, with 60 clinical cases (13 deaths) diagnosed from villages where only a total of four cases had been documented in the previous 50 years [7]. Determinants of this outbreak have remained unexplained. Investigations led to the discovery and first worldwide description of asymptomatic VL cases [8]; indeed, chronic L. infantum asymptomatic infections have been shown thereafter to be widespread in southern European countries [9]. Very recently (2010-2012) another localised VL outbreak with more than 100 cases has occurred near Madrid, Spain, where major determinants have been identified in environmental changes and the unusual role of hares as reservoir hosts [10].

Between 1989 and 2009, Italy experienced an increase in the number of VL cases over a baseline of about 10 to 30 cases reported annually since the 1950s. The number of cases peaked in 2000 and 2004, when more than 200 cases/year were observed and thereafter began to decline. The bell shape of the epidemic wave, of 20-years in width, and the substantial lack of aggressive control programmes that could justify the general decline of $V L$ incidence, strongly resemble in trend the historical $L$. donovani multi-annual epidemics, although the number of cases involved is dramatically lower. Indeed, there is still not enough knowledge on the epidemiology of $L$. infantum, and our surveillance data show that epidemics can present in a different way than expected. In this paper, we have retrospectively analysed both published and unpublished 
information collected by our unit within the frame of VL epidemiological research and surveillance in Italy, in order to identify possible determinants explaining the recent trend of the disease in the country.

\section{Methods}

\section{Study design}

Human VL data collection was based on available notifications, review of published literature and unpublished information. Basic data for all patients included at least the year of diagnosis and the patient's residence at the level of the first administrative unit (region). More detailed information regarding selected patient groups were analysed from both published and unpublished data collected by our unit since 1982 . Canine reservoir data collection was based on the review of published literature and unpublished information available at our unit or obtained from public veterinary reference centres in Italy.

\section{Human data}

VL is a compulsory notifiable disease in Italy since 1956. Diagnosed cases are recorded at the provincial local health units to which hospitals belong; notifications are gathered at regional level and subsequently centralised at the Ministry of Health. However under/ late reporting may occur from some provinces or when VL is diagnosed in patients suffering from associated conditions such as human immunodeficiency virus (HIV) co-infection, or organ transplant.

Online notifications centralised at the Italian Ministry of Health (last update: 2009 [11]) and notifications available upon request at the regional or provincial local health units registries until 2012, were integrated with information stored in the database of our unit at Istituto Superiore di Sanità, which holds detailed patient's information from an average of $50 \%$ (annual range: $43-69 \%$ ) of cases annually notified in Italy, thanks to centralised VL diagnosis and medical surveillance activities performed by our laboratories [12]. Diagnosis in clinically-suspected patients was routinely performed on clinical samples sent by hospitals (paediatrics, internal medicine, and infectious diseases wards) from throughout the country. Serology (immunofluorescent antibody test, IFAT) and microscopy, culture and polymerase chain reaction (PCR) (the last technique being employed routinely since 1997) on bone-marrow and/or peripheral blood (buffy coat) material were used in combination as recommended by World Health Organization guidelines $[13,14]$. If VL was confirmed, relevant information on patients was obtained, which included demographic data, place of hospitalisation, putative place of infection, concomitant/underlying conditions, drug regimens used and post-therapy results. Isolation of parasites from VL clinical samples was also routinely performed, resulting in a large collection of 871 Leishmania strains from different Italian areas and patient categories. Parasites were identified by multi-locus enzyme electrophoresis
(MLEE) following the standard Montpellier (MON) nomenclature [15], and/or by molecular techniques suitable for $L$. infantum identification including PCRrestriction fragment length polymorphism (RFLP) of a repetitive DNA sequence [13] or by internal transcribed spacer 1 (ITS-1) nested-PCR-RFLP [16].

\section{Data on canine leishmaniasis}

Besides the analysis of published literature, that was employed to map historical canine leishmaniasis (CanL) seroprevalence rates, recent information on diagnosis in dogs was obtained for the period from 2005 to 2011 (and updated in 2012) from the network of 10 institutes for zooprophylaxis as well as from six collaborating faculties of veterinary sciences. The main objective, to be accomplished in the frame of the European seventh framework programme (FP7) project EDENext, consisted in the country-wide mapping of communes (lowest administrative level) with undisputable presence of autochthonous CanL as an indicator of endemic transmission.

\section{Results}

General trend of visceral leishmaniasis in Italy Laboratory-confirmed recorded cases of VL in Italy from 1982 to 2012 are shown in Figure 1. Between 1950 and 1981, the number of annual cases ranged between 10 and 30 cases/year [17]. Starting from 1989 (44 (ases), a steady increase was observed with a peak in 2000 and again in 2004 involving 215 and 204 cases, respectively. A progressive decline in the annual number of cases occurred during the subsequent eight years, reaching on average one third of peak cases in the period between 2010 and 2012.

\section{Major components of the epidemic trend}

Three major components of the epidemic curve were identified including (i) an outbreak involving parts of three provinces of the Campania region, (ii) an HIV-VL co-infection epidemic and (iii) a generalised increase of VL in regions of peninsular and insular Italy.

\section{Epidemic involving parts of three} provinces of the Campania region Starting from 1989, an increase of VL cases occurred, resulting in not only major hospitals with experience in VL management, receiving cases. As some cases were misdiagnosed and/or received inappropriate treatment [18], a dedicated surveillance was implemented in the Campania region consisting in VL testing centralised at Istituto Superiore di Sanità in Rome. The surveillance disclosed the beginning of an epidemic trend that, from 1989 to 1990 , involved areas of three provinces, namely Naples (Vesuvius area), Caserta (inland area) and Salerno (coastal area). Case clusters were identified in several villages or peri-urban districts reaching an incidence of two VL cases/1,000 population in some years. A striking feature was that this epidemic was unrelated to HIV co-infections [19], which were occurring also in 
Distribution of annual number of laboratory-confirmed visceral leishmaniasis cases in Italy, 1982-2012 (n=3,122)

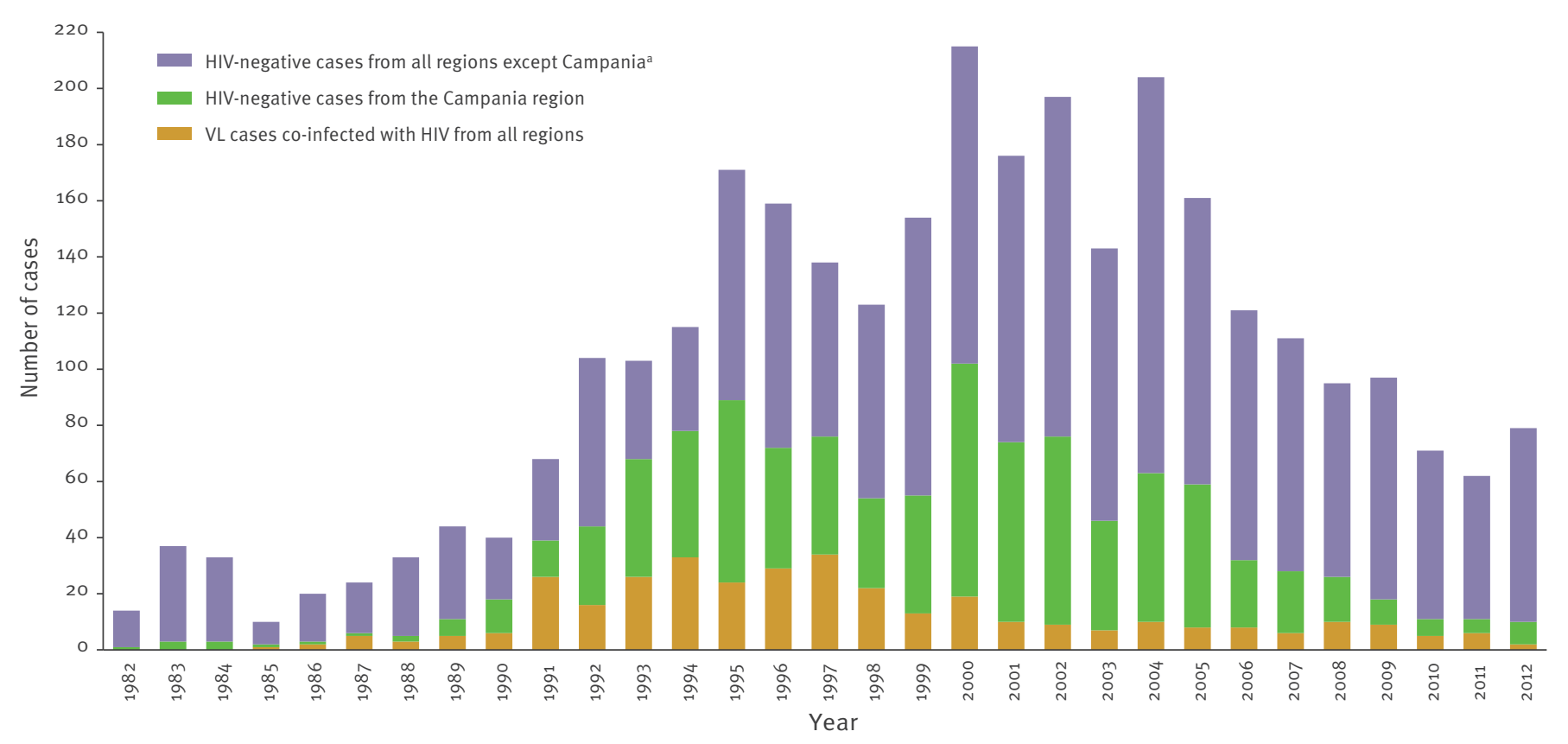

HIV: human immunodeficiency virus; VL: visceral leishmaniasis.

a Each year the largest part (>90\%) of the HIV-negative cases from all regions other than Campania, included patients from regions of peninsular and insular Italy, the remaining cases being from regions of northern continental Italy.

the form of an epidemic in other Italian regions in the same period. Parasite identification carried out on 225 strains up to the year 2003, revealed that about half of the cases (110 strains) were due to a novel zymodeme of $L$. infantum (MON-72) found also in dogs and in phlebotomine vectors from Campania foci $[20,21]$. The analysis of clinical records did not suggest any particular virulence of this zymodeme as compared to the commonest agent of Mediterranean VL, zymodeme MON-1.

Altogether 789 cumulative cases were diagnosed during the long outbreak period from 1989 to 2008 (Figure 1). After a peak of 83 cases in 2000 , the annual number of cases dropped slowly to less than 10 cases/ year after 2009. Since, the disease has become sporadic in all age groups, including children, and disappeared from most of the former 'outbreak villages and districts' although it emerged with a few cases in the remaining two provinces of Campania region (Avellino and Benevento).

\section{Human immunodeficiency virus-visceral}

leihmaniasis co-infection epidemic

A dedicated surveillance network centralised at World Health Organization headquarters, Geneva, was established after a meeting held at Istituto Superiore di Sanità, Rome, in 1994. From the beginning, our unit participated in the network by reporting information collected through a national monitoring system [22].
First HIV-VL cases were recorded in 1985. A sharp increase in the annual number of cases was observed in 1991 followed by two peaks in 1994 (33 cases) and 1997 (34 cases). The introduction and generalised use of highly active antiretroviral therapy (HAART) for HIV infection at a time between 1997 and 1998 resulted in a clear decrease in the incidence of HIV-VL co-infections. Since 2001, a plateau of about 10 cases/year or less has been observed until present (Figure 1). Cases were recorded from several regions of Italy, with higher incidences in Sicily, Lombardy and Latium. In all the epidemic phases, clinical and epidemiological features of HIV-VL were very similar to those of other Mediterranean affected countries, such as Spain and France, and have been reviewed for all cases recorded up to 2006 [23]. VL relapses, which occurred invariably in acquired immunodeficiency syndrome (AIDS) individuals before the HAART era, became less frequent in these patients following the epidemic decline. Of note, these relapses still characterise a number of coinfected patients, resulting in a significant impact on the public healthcare system.

The characterisation of $L$. infantum strains isolated from HIV-patients in Italy until 1998 has been extensively reviewed [15], and shows the high level of genetic polymorphism of these strains. In subsequent years, 51 new strains were isolated and identified by MLEE and/ or molecular methods. Altogether, 206 strains (163 
Yearly composition of zymodemes of Leishmania infantum isolated from human immunodeficiency virus-infected patients with visceral leishmaniasis (primary infection), Italy, 1986-2007 ( $\mathrm{n}=126)$

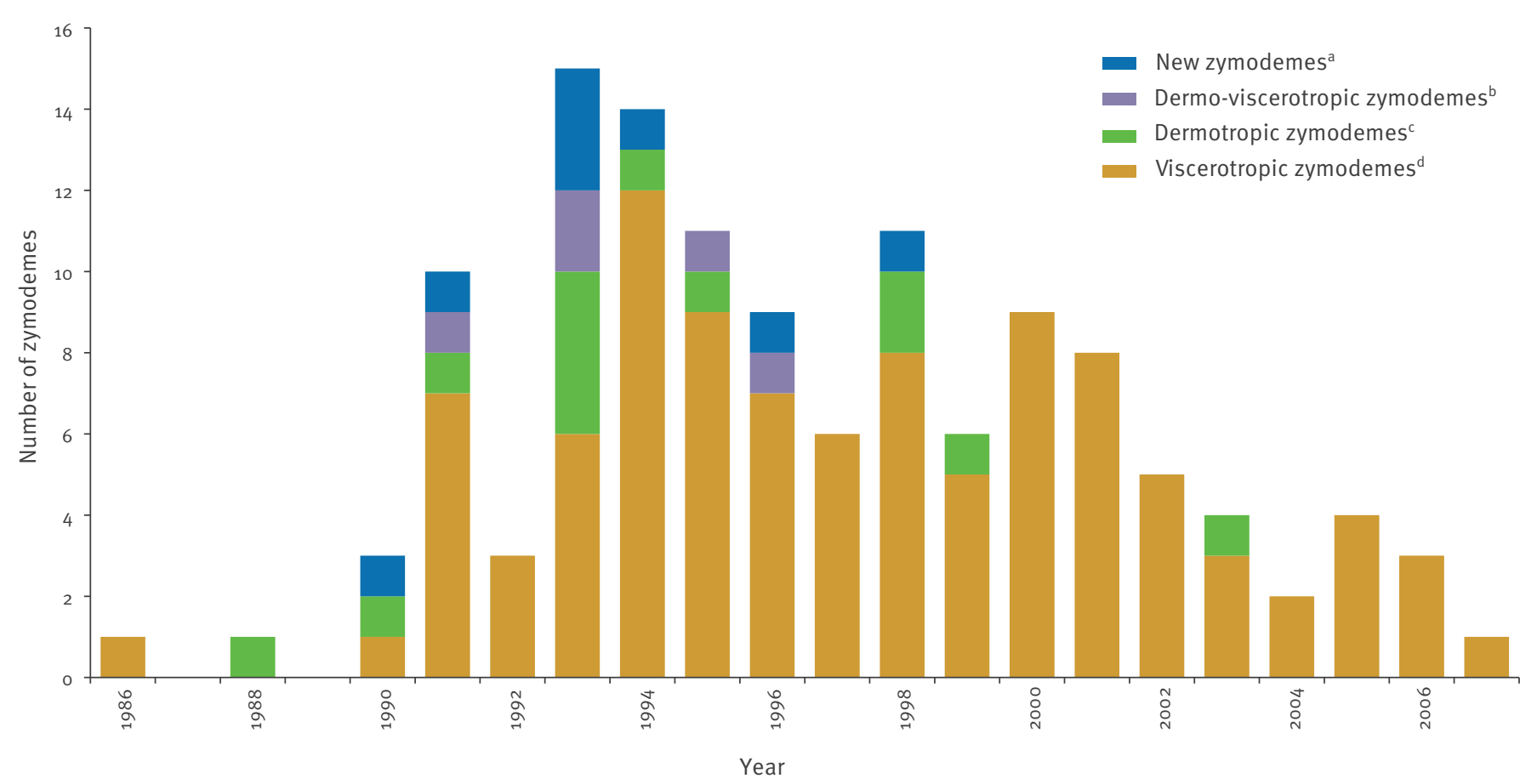

MON: Montpellier nomenclature.

a New zymodemes are represented by MON-136, MON-185, MON-188, MON-190, MON-201, MON-228, MON-183 var. MDH100 and MON-189 var. NH140 (1 strain each).

b Dermo-viscerotropic zymodemes are represented by MON-34 (4 strains) and MON-80 (1 strain).

c Dermotropic zymodemes are represented by MON-11 (1 strain), MON-24 (6 strains), MON-29 (2 strains), MON-78 (2 strains) and MON-189 (1 strain).

d Viscerotropic zymodemes are represented by MON-1 (102 strains) and MON-72 (1 strain).

from primary infections, the remaining from disease relapses) were typed from cases which occurred during the 1986 to 2007 period. L. infantum was confirmed as the main agent responsible of the co-infections, whereby only one case of co-infection with $L$. donovani was recorded and this case was imported. The annual composition of zymodemes from primary infections was more diversified in the group of strains obtained before HAART therapy introduction than in the group obtained after HAART. The pre-HAART group was composed of 84 strains collected up to 1998, and the postHAART group included 42 strains typed between 1999 and 2007 (MLEE analysis data are not available after this year). As shown in Figure 2, the former group consisted of 16 zymodemes, dominated by the viscerotropic MON-1 (6o/84, 71\%). The remaining zymodemes, represented by one to six strains each, showed variations in one to four enzyme patterns and were identified as the already known dermotropic $(n=4)$, viscerotropic $(n=1)$ and dermo-viscerotropic $(n=2)$ zymodemes, and eight new zymodemes never isolated from HIVnegative leishmaniasis patients. Conversely, the postHAART group consisted only in three zymodemes, mainly composed by MON-1 (40/42, 95\%) and by two known dermotropic zymodemes (1 strain each). The difference in zymodeme composition between the two strain groups scored as difference in the proportion of strains with the MON-1 zymodeme 'MON-1' versus those with zymodemes other than MON-1 'non-MON-1' in each group (60 vs 24 strains, and 40 vs 2 strains in pre-HAART and post-HAART period, respectively) was highly significant (Fisher exact test, P<0.001). Of note, HIV-VL cases were referred to our unit from the same hospitals distributed throughout the country during the whole period from 1986 to 2007. These findings suggest that HAART therapy, while conferring an immune restoration, may also operate a negative selection towards less virulent agents of $\mathrm{VL}$ in treated immunocompromised patients.

\section{Generalised increase of visceral leishmaniasis} in regions of peninsular and insular Italy Taking the Campania region aside, the peninsular regions of the Tyrrhenian, Ionian and Adriatic coast, as well as Sicily and Sardinia islands (all historically endemic for $\mathrm{VL}$ ) showed a generalised increase of VL in HIV-negative individuals, which was remarkable from 1995 (79 cases) to 2007 (68 cases) with a peak of 130 
Visceral leishmaniasis cases recorded among human immunodeficiency virus-negative individuals in regions other than the Campania region, Italy, 1995-2007 (n=1,032)

\begin{tabular}{|l|c|c|c|c|c|c|c|c|c|c|c|c|c|}
\hline Region & 1995 & 1996 & 1997 & 1998 & 1999 & 2000 & 2001 & 2002 & 2003 & 2004 & 2005 & 2006 & 2007 \\
\hline Liguria & 8 & 6 & 1 & 1 & 6 & 5 & 6 & 3 & 5 & 4 & 6 & 3 & 9 \\
\hline Tuscany & 1 & 3 & 3 & 2 & 2 & 5 & 4 & 9 & 8 & 10 & 6 & 4 & 10 \\
\hline Latium & 13 & 18 & 15 & 17 & 16 & 27 & 23 & 21 & 23 & 23 & 29 & 22 & 23 \\
\hline Abruzzo & 0 & 1 & 2 & 2 & 2 & 5 & 0 & 1 & 2 & 5 & 6 & 3 & 0 \\
\hline Apulia & 5 & 11 & 10 & 2 & 12 & 11 & 17 & 6 & 4 & 7 & 1 & 6 & 2 \\
\hline Calabria & 10 & 4 & 6 & 4 & 6 & 1 & 7 & 7 & 2 & 6 & 5 & 9 & 6 \\
\hline Sardinia & 7 & 13 & 2 & 5 & 4 & 8 & 5 & 9 & 5 & 5 & 2 & 4 & 3 \\
\hline Sicily & 33 & 27 & 17 & 24 & 26 & 36 & 27 & 52 & 29 & 43 & 25 & 18 & 7 \\
\hline
\end{tabular}

Visceral leishmaniasis cases recorded in human immunodeficiency virus-negative individuals in the most endemic regions of peninsular and insular Italy - other than the Campania region - in 1995-2007, corresponding to the period of highest incidence among this population. Cells reporting the two highest values of numbers of cases recorded by region are shaded in grey, showing the uneven distribution of the hot spots.

cases in 2004 (Figure 1). Case analysis showed that such increase was not associated to large case clusters in particular geographical areas, but rather to a generalised spreading of transmission foci involving one or more endemic regions depending on the years (Table) and affecting different provinces.

Minor component of the trend: visceral leishmaniasis in northern continental Italy Starting from 1990, regions from northern continental Italy traditionally considered free from Leishmania transmission had become focally endemic for $L$. infantum. This was definitely shown through active investigations involving canine serosurveys and phlebotomine sandfly monitoring during the 2003 to 2006 period in six regions (Piedmont, Valle d'Aosta, Lombardy, Veneto, Trentino Alto-Adige and Friuli-Venezia Giulia) [24]. With regard to human VL, cases had been regularly diagnosed between 1982 and 2012 in residents from all the above regions (a range of 5-15 cases/year, including HIV-co-infected individuals) mainly from cities of Lombardy and Piedmont. Travel histories were not available for cases notified to the Ministry of Health and most of them should be considered as imported from highly endemic southern regions of Italy or other Mediterranean counties as the consequence of travels during summer holidays. On the other hand, a few indisputable cases classified as autochthonous have been identified during the above active investigations [24] and also diagnosed thereafter by our laboratories, consisting of HIV-negative individuals from newly endemic areas of Piedmont, Lombardy and Veneto.

\section{Individual factors: age and}

\section{associated conditions}

To investigate further on determinants of the Italian epidemic trend, we analysed the age distribution and underlying/concomitant conditions associated to VL. Full data were available for patients diagnosed from 1987 to 2005 , thus representing both pre- and late epidemic phases. Records from 1,296 patients were considered. The resulting population is shown in Figure 3 , distributed by arbitrarily 'VL-driven' age groups. 755 cases (about $60 \%$ ) were adults (>17 years of age), however infants ( $<2$ years-old) were the most numerous homogeneous group (329 cases; $25 \%$ of all cases, $61 \%$ of paediatric ones, i.e. 0-16 years-old). Cases in preschool and young school children (3-6 yearsold) and in children grouped with young adolescents (7-16 years-old) were considered separately to show the relatively decreasing risk for VL associated to age increase in childhood (indeed the two age-groups have 100 cases each, yet the 3-6 years-old group spans only a total of 4 years of age, compared to 10 years spanned by the 7-16 years-old group). Adults and middle aged individuals (17-50 years-old) were considered as a single group because these formed the age group at higher risk for HIV infection, and accounted for 575 patients ( $44 \%$ of all cases, $76 \%$ of adults $>17$ years-old). The age group between 51 and 70 years accounted for 140 cases, and the elderly group (>70 years-old, including several individuals over 80 years of age) for 40 cases.

Patients without any recorded underlying/concomitant conditions represented the greatest majority of the population, including 1,049 cases ( $81 \%) .179$ HIV coinfected patients were recorded, representing $14 \%$ of total cases $(n=1,296)$ and $31 \%$ of the $17-50$ age-group patients $(n=575)$. One hundred and ninety-seven (99\%) of these HIV patients were adults. Finally, associated conditions other than HIV infection were recorded in 68 patients representing $5 \%$ of total cases. Fifty-seven of the 68 patients were adults, representing $8 \%$ (57/755) 
of any adults. Twenty-three underlying/concomitant conditions other than HIV infection were recorded. They were single in 54, or associated in 14 patients. The most frequent conditions were organ transplantation (14 cases), hepatitis C and liver cirrhosis (12 cases each), pregnancy (9 cases), leukaemia ( 7 cases), lymphoma (5 cases), hepatitis B and systemic lupus erythematosus ( 4 cases each), thymoma and diabetes (3 cases each), and haemophagocytosis ( 2 cases). Conditions found in one case each were chronic glomerulonephritis, multiple sclerosis, pneumonia, primitive $\mathrm{CD}_{4}$ deficit, infection with cytomegalovirus, Wegener's granulomatosis, rheumatoid arthritis, thalassaemia, splenectomy, pericarditis and chronic renal failure.

Our findings point out that even if 'physiologically less immunocompetent' groups like infants and the elderly were excluded (for a total of 369 patients), the large majority of remaining cases $(680 / 927,73 \%)$ still consisted of immunocompetent individuals for whom susceptibility to VL disease remains unexplained.

\section{Canine leishmaniasis data}

\section{General distribution}

CanL is widespread in southern Europe, but the highest values of predicted seroprevalence are found in the Italian peninsula. The median prevalence calculated from 377 canine serosurveys performed in Italy from
1971 through 2006, involving about 424,000 dogs, was $18 \%$ with a range of $11-21 \%$ in different decennia when the surveys were performed [25]. Of $494 \mathrm{~L}$. infantum canine strains characterised by MLEE, the greatest part belong to zymodeme MON-1 (457 strains), found distributed throughout the country; a large group of strains $(n=36)$ from the Campania region was found to belong to the variant zymodeme MON-72. These homogeneous groups of canine zymodemes did not reflect the elevated zymodeme polymorphism detected among the agents of human disease (both VL and cutaneous leishmaniasis), leaving the role of dogs as reservoir for all $L$. infantum zymodemes endemic in Italy unexplained.

A 2005 to 2012 data mapping on the presence of autochthonous CanL among approximately 8,100 Italian communes is shown in Figure 4. Although still incomplete because of the lack of investigations in several areas, about 2,700 communes (33\%) have been found endemic for CanL so far.

\section{Canine reservoir in the Campania region}

Cant has long been known to be endemic at high prevalences in the Campania region. In 1999, one year before the peak of $83 \mathrm{VL}$ cases recorded in the region, the local institute for zooprophylaxis had examined 1,675 dogs and found 251 seropositives at IFAT titres $>1 / 80$, corresponding to a seroprevalence of $15 \%$ [26].

\section{FIGURE 3}

Age distribution and underlying/concomitant conditions recorded in 1,296 patients affected by visceral leishmaniasis in Italy, 1987-2005

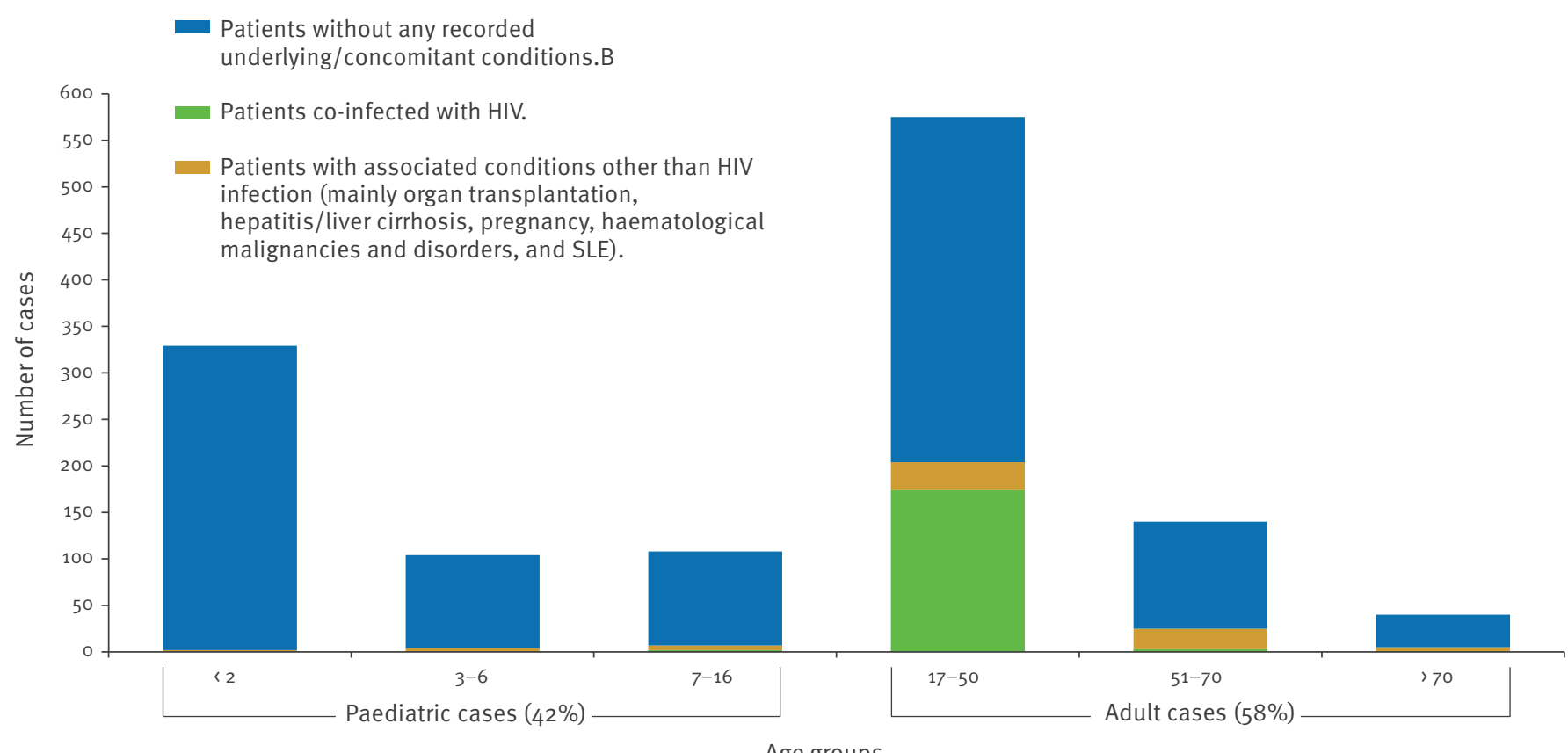

HIV: human immunodeficiency virus; SLE: systemic lupus erythematosus. 
In the 2005 to 2009 period, corresponding to the drop of VL incidence in that region, the same Institute reported 9,723/70,557 seropositive dogs at the same IFAT titre range, corresponding to a seroprevalence of $14 \%$ i.e. very similar to the value recorded in 1999 (2010, data from Istituto Zooprofilattico Sperimentale del Mezzogiorno, Portici, Naples). These findings suggest that canine seroprevalence rates cannot per se explain the epidemic trends of human VL.

\section{Discussion}

At country-wide level, leishmaniasis epidemics may consist of an increase of disease cases over the expected baseline in the general population throughout the territory, or represent the additive outcome of different outbreaks occurring in particular areas and/ or affecting specific groups of individuals. Epidemic trends have a typical bell shape where the increasing slope represents the natural epidemic onset, whereas the decreasing slope may either be natural or due to aggressive human interventions to control the disease. Our analysis indicates that the VL epidemic in Italy was a complex phenomenon, in which more than one component was represented: (i) an outbreak involving infants and immunocompetent individuals in parts of the Campania region, that appears to have declined naturally; (ii) a second outbreak affecting HIV-infected individuals throughout the country, that declined via the use of HAART therapies, which also probably applied a negative selection pressure on less virulent Leishmania genotypes originally involved in the epidemic. Hence, the contribution of the HIV-VL epidemic to the general VL trend in Italy has been limited in time, so that the two trends peaked and started to decline in different years. As mentioned above, the HIV-VL trend did not affect greatly the Campania VL trend, since the occurrence of co-infected cases in this region was negligible.; (iii) a generalised increase of cases due to disease spreading within traditionally endemic areas (a major contribution in cases) as well as the appearance of cases in previously non-endemic areas (a minor contribution in cases).

While the appearance of autochthonous VL in northern continental Italy could reasonably be explained by the de novo colonisation of these areas by phlebotomine vectors along with the frequent importation of Leishmania-infected dogs from the endemic south [24], and the generalised increase of cases in endemic areas by an increase in transmission potential due to changes in vector density, both the onset and the natural decline of the outbreak in the Campania region, traditionally endemic for $\mathrm{VL}$, will probably remain unexplained. One reason for the decline could be searched in the immunity levels against Leishmania acquired by the population during the epidemic peaks, however this hypothesis is difficult to verify because of the sporadic nature of the disease that would require large population cohorts to be examined prospectively.
FIGURE 4

Map (ArcView GIS 10) showing the distribution of communes (in blue) where autochthonous cases of canine leishmaniasis have been recorded, Italy, 2005-2012

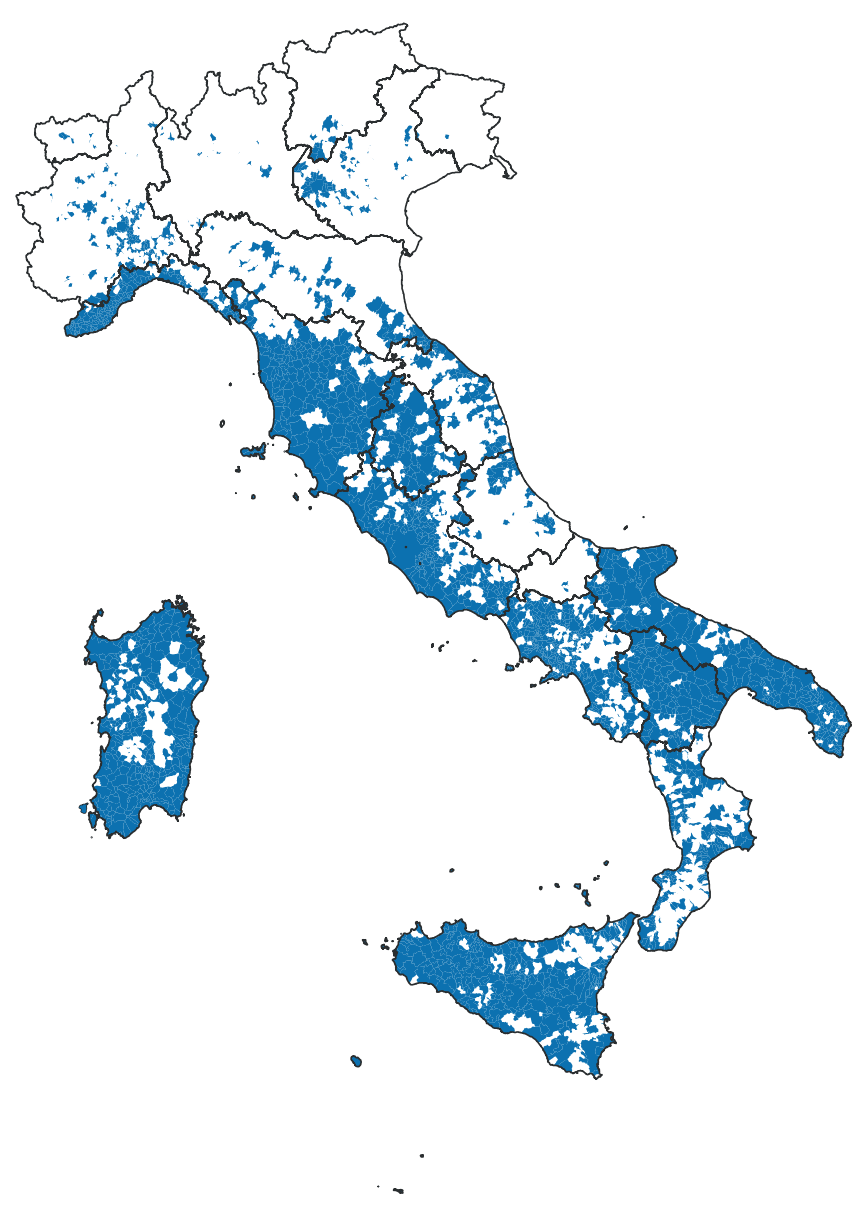

GIS: geographic information system.

Other investigated epidemiological compartments of the zoonotic VL cycle, the canine reservoir and phlebotomine vectors, did not help in elucidating the causes of the Italian epidemic. First of all, no aggressive programmes to control the increase of VL, that may justify the general drop in incidence, were ever put in place at country level. Indeed, only some regions have implemented guidelines and rules to monitor infections in pets and/or kennelled stray dogs, recommending drug treatment of infected animals and the use of topical insecticides against sandflies. However, as far as pet dogs are concerned, both measures were left at the owners' expenses resulting in the limited coverage of the dogs to control. Because canine topical insecticides have an impact on CanL incidence only after repeated mass use [27] and drug treatments may have some efficacy in decreasing transmission only when administered during the sandfly season, the measures actually undertaken were most probably not very effective in reducing the Leishmania transmission potential. 
Incidence of CanL may vary considerably within endemic areas, with focal distribution. To date there is no clear evidence for a direct association of CanL prevalence values and incidence of human VL disease in a given territory. Indeed, several examples are available in literature where very elevated prevalences recorded in dogs did not result in human VL cases at all [28]. It implies that although dogs are efficient sentinel hosts for the Leishmania transmission in a given territory, and hence the finding of autochthonous CanL cases in previously non-endemic areas can be of value to predict the occurrence of human cases, the prevalence rate of canine infections does not appear an useful parameter to explain determinants of human VL trends in endemic areas, like we observed in Campania region.

With regards to the phlebotomine vectors, Italy is endemic for four Phlebotomus species proven to transmit $L$. infantum, $P$. perniciosus being the most widespread and efficient vector. A recent atlas based on validated bibliographical records on phlebotomine sandflies from 1985 to 2009 reported the presence of this species in 134 of approximately 8,100 Italian communes throughout the country. Among the other vector species, $P$. perfiliewi was recorded in 50 communes of peninsular Italy, $P$. neglectus in 41 communes from southern and northern, but not from central Italy, and P. ariasi in four communes at the French border [29]. Hence, considering the huge disproportion with available CanL data, information on phlebotomine vector distribution in Italy is still largely incomplete. On the other hand, like for CanL, to date there is no clear evidence for a direct association of phlebotomine vector presence and the incidence of human VL in a given territory. Indeed, many parameters necessary to a robust definition of vectorial capacity in phlebotomine species, such as standardised density measurements, dog- versus man-biting rates, number and frequency of blood meals/ovodepositions, infected vs infectious sandfly ratio, are still lacking from the scientific literature worldwide. Hence, while the presence of competent vectors in a given territory can be predictive for the occurrence of human VL when CanL cases are found too, current entomological data are still of low informative value for the analysis of epidemic VL trends.

\section{Acknowledgements}

This study was funded by EU grant FP7-261504 EDENext and is catalogued by the EDENext Steering Committee as EDENext162 (http://www.edenext.eu). The contents of this publication are the sole responsibility of the authors and do not necessarily reflect the views of the European Commission.
References

1. Antoniou M, Haralambous C, Mazeris A, Pratlong F, Dedet JP, Soteriadou K. Leishmania donovani leishmaniasis in Cyprus. Lancet Infect Dis. 2009;9(2):76-7.

http://dx.doi.org/10.1016/S1473-3099(09)70004-0

2. Ashford RW, Bettini S. Ecology and epidemiology: Old World. In: Peters W, Killick-Kendrick, R, editors. The leishmaniases in biology and medicine. London: Academic Press; 1987. p. 365-424.

3. Dye C, Wolpert DM. Earthquakes, influenza and cycles of Indian kala-azar. Trans R Soc Trop Med Hyg. 1988;82(6):84350.

http://dx.doi.org/10.1016/0035-9203(88)90013-2

4. Seaman J, Mercer AJ, Sondorp E. The epidemic of visceral leishmaniasis in western Upper Nile, southern Sudan: course and impact from 1984 to 1994. Int J Epidemiol. 1996;25(4):86271. PMid:8921468

5. Herrero M, Orfanos G, Argaw D, Mulugeta A, Aparicio P, Parre-o $F$, et al. Natural history of a visceral leishmaniasis outbreak in highland Ethiopia. Am J Trop Med Hyg. 2009;81(3):373-7. PMid:19706898

6. Dujardin JC, Campino L, Ca-avate C, Dedet JP, Gradoni L, Soteriadou K, et al. Spread of vector-borne diseases and neglect of Leishmaniasis, Europe. Emerg Infect Dis. 2008;14(7):1013-8.

http://dx.doi.org/10.3201/eid1407.071589 PMid:18598618 PMCid:PMC2600355

7. Pampiglione S, La Placa M, Schlick G. Studies on mediterranean Leishmaniasis. I. An outbreak of visceral leishmaniasis in Northern Italy. Trans R Soc Trop Med Hyg. 1974;68(5):349-59.

http://dx.doi.org/10.1016/0035-9203(74)90148-5

8. Pampiglione S, Manson-Bahr PE, Giungi F, Giunti G, Parenti A, Canestri Trotti G. Studies on Mediterranean leishmaniasis. 2. Asymptomatic cases of visceral leishmaniasis. Trans R Soc Trop Med Hyg. 1974;68(6):447-53. http://dx.doi.org/10.1016/0035-9203(74)90067-4

9. Michel G, Pomares C, Ferrua B, Marty P. Importance of worldwide asymptomatic carriers of Leishmania infantum (L. chagasi) in human. Acta Trop. 2011;119(2-3):69-75. http://dx.doi.org/10.1016/j.actatropica.2011.05.012 PMid:21679680

10. Molina R, Jiménez MI, Cruz I, Iriso A, Martín-Martín I, Sevillano 0 , et al. The hare (Lepus granatensis) as potential sylvatic reservoir of Leishmania infantum in Spain. Vet Parasitol. 2012;190(1-2):268-71.

http://dx.doi.org/10.1016/j.vetpar.2012.05.006 PMid:22677135

11. Italian Ministry of Health. [Accessed 17 Jul 2013]. Available from: http://www.salute.gov.it

12. Gradoni L, Gramiccia M, Scalone A. Visceral leishmaniasis treatment, Italy. Emerg Infect Dis. 2003;9(12):1617-20. http://dx.doi.org/10.3201/eido912.030178 PMid:14720406 PMCid:PMC3034325

13. Cascio A, Calattini S, Colomba C, Scalamogna C, Galazzi M, Pizzuto $\mathrm{M}$, et al. Polymerase chain reaction in the diagnosis and prognosis of Mediterranean visceral leishmaniasis in immunocompetent children. Pediatrics. 2002;109(2):E27. http://dx.doi.org/10.1542/peds.109.2.e27 PMid:11826237

14. World Health Organization (WHO). Control of the leishmaniases. Tech Rep Ser 949. Geneva: WHO; 2010. 186 p. Available from: http://whqlibdoc.who.int/trs/WHO_TRS_949 eng.pdf

15. Gramiccia M. The identification and variability of the parasites causing leishmaniasis in HIV-positive patients in Italy. Ann Trop Med Parasitol. 2003;97 Suppl 1:65-73. http://dx.doi.org/10.1179/000349803225002543 PMid:14678634

16. Schönian G, Nasereddin A, Dinse N, Schweynoch C, Schallig $\mathrm{HD}$, Presber W, et al. PCR diagnosis and characterization of Leishmania in local and imported clinical samples. Diagn Microbiol Infect Dis. 2003:47(1):349-58. http://dx.doi.org/10.1016/S0732-8893(03)00093-2

17. Istituto Centrale di Statistica. Annuario di Statistiche Sanitarie. Roma: Abete Edizioni; 1951-1981.

18. Gradoni L, Pizzuti R, di Martino L, Gramiccia M, Pempinello R, Gaeta GB, et al. The epidemiology and surveillance of visceral leishmaniasis in the Campania region of Italy. The value of zymodeme typing. Epidemiol Infect. 1993;111(2):297-306. http://dx.doi.org/10.1017/S0950268800057009 PMid:8405156 PMCid:PMC2271383

19. Gradoni L, Pizzuti R, Scalone A, Russo M, Gramiccia M, di Martino L, et al. Recrudescence of visceral leishmaniasis 
unrelated to HIV infection in the Campania region of Italy.

Trans R Soc Trop Med Hyg. 1996;90(3):234-5.

http://dx.doi.org/10.1016/So035-9203(96)90226-6

20. Gramiccia M, Gradoni L, di Martino L, Romano R, Ercolini D.

Two syntopic zymodemes of Leishmania infantum cause human

and canine visceral leishmaniasis in the Naples area, Italy.

Acta Trop. 1992 Apr;50(4):357-9.

http://dx.doi.org/10.1016/0001-706X(92)90072-6

21. Maroli M, Gramiccia M, Gradoni L, Troiani M, Ascione R.

Natural infection of Phlebotomus perniciosus with MON 72

zymodeme of Leishmania infantum in the Campania region of

Italy. Acta Trop. 1994;57(4):333-5.

http://dx.doi.org/10.1016/0001-706X(94)90079-5

22. Gradoni L, Scalone A, Gramiccia M, Troiani M. Epidemiological surveillance of leishmaniasis in HIV-1-infected individuals in Italy. AIDS. 1996;10(7):785-91.

http://dx.doi.org/10.1097/00002030-199606001-00014 PMid:8805871

23. Alvar J, Aparicio P, Aseffa A, Den Boer M, Ca-avate C, Dedet JP, et al. The relationship between leishmaniasis and AIDS: the second 10 years. Clin Microbiol Rev. 2008;21(2):334-59.

http://dx.doi.org/10.1128/CMR.00061-07

PMid:18400800 PMCid:PMC2292576

24. Maroli M, Rossi L, Baldelli R, Capelli G, Ferroglio E, Genchi

$C$, et al. The northward spread of leishmaniasis in Italy: evidence from retrospective and ongoing studies on the canine reservoir and phlebotomine vectors. Trop Med Int Health. 2008;13(2):256-64.

http://dx.doi.org/10.1111/j.1365-3156.2007.01998.

PMid:18304273

25. Franco AO, Davies CR, Mylne A, Dedet JP, Gállego M, Ballart $C$, et al. Predicting the distribution of canine leishmaniasis in western Europe based on environmental variables. Parasitology. 2011;138(14):1878-91.

http://dx.doi.org/10.1017/So03118201100148X

PMid:21914251

26. Scalone A, De Luna R, Oliva G, Baldi L, Satta G, Vesco G, et al. Evaluation of the Leishmania recombinant K39 antigen as a diagnostic marker for canine leishmaniasis and validation of a standardized enzyme-linked immunosorbent assay. Vet Parasitol. 2002;104(4):275-85. http://dx.doi.org/10.1016/S0304-4017(01)00643-4

27. Maroli M, Gradoni L, Oliva G, Castagnaro M, Crotti A, Lubas G, et al. Guidelines for prevention of leishmaniasis in dogs. J Am Vet Med Assoc. 2010;236(11):1200-6.

http://dx.doi.org/10.246o/javma.236.11.1200

PMid:20513198

28. Bettini S, Gradoni L. Canine leishmaniasis in the Mediterranean area and its implications for human leishmaniasis. Insect Sci Appl. 1986;7: 241-5.

29. Busani L, Mughini Gras L, Romi R, Boccolini D, Severini F, Bongiorno G, et al. Zanzare, flebotomi e zecche: atlante bibliografico delle specie di interesse sanitario in Italia (1985-1989). [Mosquitoes, sand flies and ticks: bibliographical atlas of species of medical importance in Italy (1985-2009)]. Rapporti ISTISAN 12/22. Roma: Istituto Superiore di Sanità; 2012. Italian. Available from: http://www.iss.it/binary/publ/ cont/12_22_web.pdf 